\title{
HITTING TIMES AND THE RUNNING MAXIMUM OF MARKOVIAN GROWTH-COLLAPSE PROCESSES
}

\author{
ANDREAS LÖPKER, ${ }^{*}$ Eindhoven University of Technology and EURANDOM \\ WOLFGANG STADJE, ${ }^{* *}$ University of Osnabrück
}

\begin{abstract}
We consider the level hitting times $\tau_{y}=\inf \left\{t \geq 0 \mid X_{t}=y\right\}$ and the running maximum process $M_{t}=\sup \left\{X_{s} \mid 0 \leq s \leq t\right\}$ of a growth-collapse process $\left(X_{t}\right)_{t \geq 0}$, defined as a $[0, \infty)$-valued Markov process that grows linearly between random 'collapse' times at which downward jumps with state-dependent distributions occur. We show how the moments and the Laplace transform of $\tau_{y}$ can be determined in terms of the extended generator of $X_{t}$ and give a power series expansion of the reciprocal of $\mathrm{E}^{-s \tau_{y}}$. We prove asymptotic results for $\tau_{y}$ and $M_{t}$ : for example, if $m(y)=\mathrm{E} \tau_{y}$ is of rapid variation then $M_{t} / m^{-1}(t) \stackrel{\mathrm{W}}{\rightarrow} 1$ as $t \rightarrow \infty$, where $m^{-1}$ is the inverse function of $m$, while if $m(y)$ is of regular variation with index $a \in(0, \infty)$ and $X_{t}$ is ergodic, then $M_{t} / m^{-1}(t)$ converges weakly to a Fréchet distribution with exponent $a$. In several special cases we provide explicit formulae.
\end{abstract}

Keywords: Growth-collapse process; piecewise deterministic Markov process; hitting time; running maximum; asymptotic behavior; regular variation; separable jump measure

2010 Mathematics Subject Classification: Primary 60K30

Secondary 60J25; 60J75; 60F05

\section{Introduction and known results}

A Markovian growth-collapse process (MGCP) is a Markov process $\left(X_{t}\right)_{t \geq 0}$ on the state space $\mathscr{E}=[0, \infty)$ with no upward jumps and piecewise deterministic right-continuous paths. The process $X_{t}$ increases linearly with slope 1 between the jumps. Hence, it can be written in the form

$$
X_{t}=X_{0}+t-\sum_{k=1}^{N_{t}} B_{k}, \quad t \geq 0,
$$

where $\left(N_{t}\right)_{t \geq 0}$ is a state-dependent counting process and the downward jump sizes $B_{k}>0$ also depend on the current state. MGCPs can be encountered in a large variety of applications of which we mention population growth models, risk processes, stress release, neuron firing, and window sizes in transmission control protocols; they have been studied in [7], [8], [14], and [28]. They form a special class of piecewise deterministic Markov processes [6], [10], [11].

We are interested in the behavior of the first hitting time

$$
\tau_{y}=\inf \left\{t \geq 0 \mid X_{t}=y\right\}
$$

Received 5 February 2009; revision received 16 November 2010.

* Current address: Department of Economics and Social Sciences, Helmut Schmidt University, PO Box 700822, 22008 Hamburg, Germany. Email address: lopker@hsu-hh.de

** Postal address: Department of Mathematics and Computer Science, University of Osnabrück, 49069 Osnabrück, Germany. Email address: wolfgang@mathematik.uos.de 
of the level $y \geq 0$ and in the running maximum process

$$
M_{t}=\sup \left\{X_{s} \mid 0 \leq s \leq t\right\} .
$$

Note that $X_{\tau_{y}}=X_{\tau_{y}-}=y$ almost surely (a.s.). The main objective of this paper is to evaluate the Laplace transform $\mathrm{E}^{-s \tau_{y}}$ and the moments of $\tau_{y}$ for the introduced growth-collapse model, in particular the function $m(y):=\mathrm{E} \tau_{y}$, and to derive a quite general result for the convergence of $M_{t}$.

More formally, let $T_{1}, T_{2}, \ldots$ denote the times of the successive collapses (jumps) of the MGCP, and let $\lambda(\cdot)$ be the jump intensity of the process, so that the probability of a jump during $(t, t+h]$ given that $X_{t}=x$ is $\lambda(x) h+o(h)$ as $h \rightarrow 0$. The probability of a jump from $x$ into the set $[0, y]$ is given by $\mu_{x}(y)$, where $\mu_{x}$ is the distribution function of a probability measure on $[0, x)$ for each $x \in \mathcal{E}$. We assume that

- $\lambda: \& \rightarrow[0, \infty)$ is locally integrable and $\int_{0}^{\infty} \lambda(u) \mathrm{d} u=\infty$;

- $\mathrm{E}\left(N_{t}\right)<\infty$ for all $t \geq 0$.

Note that the first assumption implies that the first jump will a.s. occur in finite time. Let $\mathrm{P}_{x}$ and $\mathrm{E}_{x}$ denote the conditional probability and expectation given that $X_{0}=x$. It is easy to see that, under $\mathrm{P}_{x}$, the first hitting time of level $y>x$ has the same distribution as $\tau_{y}-\tau_{x}$ under $\mathrm{P}_{0}$, and that $\tau_{x}$ and $\tau_{y}-\tau_{x}$ are independent under $\mathrm{P}_{0}$. Hence, we assume from now on that $X_{0}=0$ and write $\mathrm{P}$ instead of $\mathrm{P}_{0}$. The process $X_{t}$ can also be viewed as a regenerative process if we define cycles as the times between successive visits to some fixed recurrent state $z \in[0, \infty)$. Let $C_{k}$ denote the length of the $k$ th cycle, where the first cycle starts at time $C_{0}=\tau_{z}$. Let $S_{k}=\sum_{i=0}^{k} C_{i}$, and let $K(t)$ denote the number of the current cycle at time $t$. Then $S_{K\left(\tau_{y}\right)-1} \leq \tau_{y}=S_{K\left(\tau_{y}\right)-1}+\bar{\tau}_{y}$, where $\bar{\tau}_{y}$ is distributed as the first hitting time of level $y$, starting from $z$ and given that the process stays above $z$. Renyi's theorem states that if $\mu_{C}=\mathrm{E}\left(C_{1}\right)<\infty$ then

$$
\frac{\mathrm{P}\left(K\left(\tau_{y}\right)=1\right)}{\mu_{C}} S_{K\left(\tau_{y}\right)-1} \stackrel{\mathrm{w}}{\rightarrow} Z \quad \text { as } y \rightarrow \infty,
$$

where ' $\stackrel{\mathrm{w}}{\rightarrow}$ ' denotes weak convergence and $Z$ is an exponential random variable with unit mean (see the extended version given as Theorem 2.4 of [17]). Let $\xi_{i}=\max \left\{X_{t} \mid t \in\left[S_{i-1}, S_{i}\right]\right\}$ denote the $i$ th cycle maximum, and let $G(y)=\mathrm{P}\left(\xi_{1} \leq y\right)=1-\mathrm{P}\left(K\left(\tau_{y}\right)=1\right)$ denote the common distribution function of the $\xi_{i}$. If $\bar{\tau}_{y}$ is small compared to $S_{K\left(\tau_{y}\right)-1}$ then we can expect that

$$
\frac{1-G(y)}{\mu_{C}} \tau_{y} \stackrel{\mathrm{w}}{\rightarrow} Z \quad \text { as } y \rightarrow \infty .
$$

The fact that this is indeed true if $X_{t}$ is ergodic is known as Keilson's theorem [18]. Propositions 2 and 3 of [8] imply that, for an MGCP, E $\tau_{y}^{n}<\infty$ for all $y \geq 0$ and all $n \in \mathbb{N}$, and that $X_{t}$ is ergodic if $\lim \sup _{x \rightarrow \infty} \lambda(x) \int_{0}^{x} \mu_{x}(y) \mathrm{d} y>1$. Moreover, it can be shown (see, e.g. [3, Proposition 4.1]) that the convergence in (1) also holds in expectation so that

$$
m(y) \sim \frac{\mu_{C}}{1-G(y)} \quad \text { as } y \rightarrow \infty .
$$

Consequently, any asymptotic result for the function $m$ is at the same time a result for the tail of $G$. It then follows from (1) and (2) that in the ergodic case

$$
\frac{\tau_{y}}{m(y)} \stackrel{\mathrm{w}}{\rightarrow} Z \quad \text { as } y \rightarrow \infty
$$


Clearly, $M_{t} \geq y$ if and only if $\tau_{y} \leq t$, so that various probabilistic properties of $\tau_{y}$ can be expressed in terms of properties of $M_{t}$, in particular via the relation $\mathrm{P}\left(\tau_{y} \leq t\right)=\mathrm{P}\left(M_{t} \geq y\right)$. Clearly, $\max _{i \leq K(t)-1} \xi_{i} \leq M_{t} \leq \max _{i \leq K(t)} \xi_{i}$, and since $K(t) \approx t / \mu_{C}$, it is to be expected that $\mathrm{P}\left(M_{t} \leq y\right)$ is close to $G(y)^{t / \mu_{C}}$. Indeed, it was shown in [25] that $\sup _{y \geq 0} \mid \mathrm{P}\left(M_{t} \leq\right.$ $y)-G(y)^{t / \mu_{C}} \rightarrow 0$ as $t \rightarrow \infty$. Hence, classical extreme value theory for independent and identically distributed variables can be applied to find possible limits of the (properly normalized) process $M_{t}$. The following results are known for general regenerative processes (see [2] and [25]). Let $\vec{G}(t)=\inf \{x: 1-G(x) \leq 1 / t\}$. A function $f:[0, \infty) \rightarrow(0, \infty)$ is called regularly varying if, for a fixed $a \in \mathbb{R}$,

$$
\frac{f(\lambda y)}{f(y)} \rightarrow \lambda^{a} \text { as } y \rightarrow \infty
$$

for all $\lambda>0$ and we then write $f \in \mathcal{R}_{a}$. Suppose that $X_{t}$ is ergodic. Then, if $1-G \in \mathcal{R}_{-a}$ for some $a>0$, we have

$$
\frac{M_{t}}{\vec{G}\left(t / \mu_{C}\right)} \stackrel{\mathrm{w}}{\rightarrow} Z_{a} \quad \text { as } t \rightarrow \infty,
$$

where $\mathrm{P}\left(Z_{a} \leq x\right)=\mathrm{e}^{-x^{-a}}$ (Fréchet distribution). If $1-G(y)=\exp \left(-\int_{0}^{y}[1 / \delta(u)] \mathrm{d} u\right)$ for some absolutely continuous function $\delta>0$ having density $\delta^{\prime}(y) \rightarrow 0$ as $y \rightarrow \infty$, then

$$
\frac{M_{t}-\vec{G}\left(t / \mu_{C}\right)}{\delta\left(\vec{G}\left(t / \mu_{C}\right)\right)} \stackrel{\mathrm{w}}{\rightarrow} Z_{G} \quad \text { as } t \rightarrow \infty,
$$

where $\mathrm{P}\left(Z_{G} \leq x\right)=\exp \left(-\mathrm{e}^{-x}\right)$ (Gumbel distribution).

In this paper we supplement the above known results with the following contributions. In Section 2 we consider the recursive sequence of equations $\mathcal{A} m_{n}=m_{n-1}, m_{0} \equiv 1$, where $\mathcal{A}$ is the extended generator of the MGCP, and show that the solution sequence (which is essentially unique and can be given in integral form) is related to the moments of $\tau_{y}$ : we have, for example, $m(y)=\mathrm{E} \tau_{y}=m_{1}(y)$ and $\mathrm{E} \tau_{y}^{2}=2\left(m_{1}(y)^{2}-m_{2}(y)\right)$. The Laplace transform of $\tau_{y}$ can be expressed in closed form (in terms of an integral involving a certain kernel) in a similar way. We also prove the power series expansion

$$
\frac{1}{\mathrm{E}^{-s \tau_{y}}}=\sum_{n=0}^{\infty} m_{n}(x) s^{n}, \quad s, x \geq 0,
$$

for the reciprocal of $\mathrm{E}^{-s \tau_{y}}$. Without assuming ergodicity of the MGCP, it can be shown (using (5)) that the relation $m_{2}(y)=o\left(m_{1}(y)\right)$ as $y \rightarrow \infty$ implies (3). In Section 3 we derive asymptotic results for the running maximum: (i) if $m(y)$ is of rapid variation, we have $M_{t} / m^{-1}(t) \stackrel{\mathrm{W}}{\rightarrow} 1$, where $m^{-1}$ denotes the inverse function of $m$; (ii) if $m(y)$ is of regular variation with index $a \in(0, \infty)$ and the MGCP is ergodic, then $M_{t} / m^{-1}(t) \stackrel{\mathrm{W}}{\rightarrow} Z_{a}$. In fact, in case (i) we even prove that all moments of $M_{t} / m^{-1}(t)$ converge to 1 . In Section 4 we present several examples. In the case of separable jump measures (i.e. $\mu_{x}(y)=v(y) / v(x), 0 \leq y \leq x$, for some function $v(x)$ ) we give various explicit results on $\tau_{y}$. Moreover, we prove that if $v$ is regularly varying with index $b$ and $x \lambda(x)$ tends to some limit $a \in(b+1, \infty]$ as $x \rightarrow \infty$, then $M_{t} / m^{-1}(t) \stackrel{\mathrm{W}}{\rightarrow} Z_{a}$ if $a<\infty$ and $M_{t} / m^{-1}(t) \stackrel{\mathrm{W}}{\rightarrow} 1$ if $a=\infty$. In applications $\lambda(x)$ is usually nondecreasing, leading to $a=\infty$; a typical case is $\lambda(x)=\lambda_{0}+\lambda_{1} x^{\beta}$ for some $\beta>0, \lambda_{0} \geq 0$, and $\lambda_{1}>0$. If $v(x)=x$, a collapse causes the cutoff of a uniform fraction of the current value, 
which can be modeled by the multiplication by a random variable that is uniform on $(0,1)$. We also present several closed-form expressions in the general case of jumps generated by means of multiplication by $(0,1)$-valued random variables. Finally, the results on regularly and rapidly varying functions that are used throughout are collected in Appendix A.

We note that instead of studying models with linear increase, we could also study MGCPs $Y_{t}$ with a more general deterministic interjump behavior, say $\mathrm{d} Y_{t}=r\left(Y_{t}\right) \mathrm{d} t$, where $r(x)$ is a positive Lipschitz continuous function. It turns out that we can easily transform $X_{t}$ into $Y_{t}$ and vice versa by means of the transformation $X_{t}=\theta\left(Y_{t}\right)$, where $\theta(x)=\int_{z}^{x} 1 / r(u) \mathrm{d} u$ measures the time the process $Y_{t}$ needs to increase from 0 to $x$. It then indeed follows that $\mathrm{d} X_{t}=\left(\mathrm{d} \theta\left(Y_{t}\right) / \mathrm{d} t\right)\left(\mathrm{d} Y_{t} / \mathrm{d} t\right)=1$ in between jumps. If $\hat{\tau_{y}}$ and $\hat{M}_{t}$ denote the first hitting times and the maximum process of $Y_{t}$, then it is easy to see that $\tau_{y}=\hat{\tau}_{\theta^{-1}(y)}$ and $M_{t}=\theta\left(\hat{M}_{t}\right)$.

\section{Integral equations and series representations}

Our derivations require the notion of the extended generator of the Markov process $X_{t}$. A measurable function $f:[0, \infty) \rightarrow[0, \infty)$ belongs to the domain of the extended generator if the process

$$
f\left(X_{t}\right)-\int_{0}^{t} g\left(X_{s}\right) \mathrm{d} s, \quad t \geq 0,
$$

is a martingale for some measurable function $g:[0, \infty) \rightarrow[0, \infty)$. In this case we write $\mathcal{A} f(x)=g(x)$ and call $\mathcal{A}$ the extended generator. Note that $\mathcal{A}$ can be multivalued.

In [11, Theorem 26.14] broad sufficient conditions for a function to be a member of the domain is given. Let $\mathcal{M}_{\mathrm{abs}}$ denote the set of absolutely continuous functions $f:[0, \infty) \rightarrow$ $[0, \infty)$ with locally bounded, nonnegative Lebesgue density $f^{\prime}(x)$. If $f \in \mathcal{M}_{\text {abs }}$ then $f$ is nondecreasing and since $X_{t} \leq t$ a.s., we have $f\left(X_{t}\right) \leq f(t)$ a.s., yielding the bound

$$
\mathrm{E} \sum_{n=1}^{N_{t}}\left|f\left(X_{T_{i}-}\right)-f\left(X_{T_{i}}\right)\right| \leq f(t) \mathrm{E} N_{t}<\infty
$$

for all $t \geq 0$. It follows from [11, Theorem (26.14)], that the functions in $\mathcal{M}_{\text {abs }}$ belong to the domain of the extended generator and that $\mathcal{A} f(x)$ is given by

$$
\mathcal{A} f(x)=f^{\prime}(x)-\lambda(x) \int_{0}^{x}(f(x)-f(y)) \mathrm{d} \mu_{x}(y),
$$

which, after applying Fubini's theorem, can be written as

$$
\mathcal{A} f(x)=f^{\prime}(x)-\lambda(x) \int_{0}^{x} f^{\prime}(y) \mu_{x}(y) \mathrm{d} y .
$$

Note that the actual domain of the extended generator may be much larger than $\mathcal{M}_{\text {abs }}$, but $\mathcal{M}_{\text {abs }}$ suffices here, since the relevant functions that appear throughout this paper belong to $\mathcal{M}_{\text {abs }}$.

In the sequel we need the kernel $K_{s}(x, y)=\lambda(x) \mu_{x}(y)+s$, where $x \geq y \geq 0$ and $s \geq 0$, and its iterates $K_{s}^{1}(x, y)=K_{s}(x, y)$ and

$$
K_{s}^{n}(x, y)=\int_{y}^{x} K_{s}(x, u) K_{s}^{n-1}(u, y) \mathrm{d} u, \quad n \geq 2 .
$$

It is straightforward to show that

$$
K_{s}^{n}(x, y) \leq(\lambda(x)+s) \frac{\left(\int_{y}^{x}(\lambda(u)+s) \mathrm{d} u\right)^{n-1}}{(n-1) !}
$$


(cf. Lemma 1 of [27]). Hence, the resolvent kernel

$$
R_{S}(x, y)=1+\sum_{k=1}^{\infty} K_{s}^{k}(x, y)
$$

is well defined and converges for all $s \geq 0$ and all $x \geq y \geq 0$. Moreover, it follows from (6) that

$$
R_{s}(x, y) \leq 1+(\lambda(x)+s) \exp \left(\int_{y}^{x}(\lambda(u)+s) \mathrm{d} u\right) .
$$

Theorem 1. 1. Let $m_{0}(x)=1$. For all $n \in \mathbb{N}$, there exists a unique solution $m_{n} \in \mathcal{M}_{\mathrm{abs}}$ of the equation $\mathcal{A}_{n}(x)=m_{n-1}(x)$ with initial condition $m_{n}(0)=0$. Moreover,

$$
m_{n}(y)=\int_{0}^{y}\left(m_{n-1}(x)+\int_{0}^{x} R_{0}(x, u) m_{n-1}(u) \mathrm{d} u\right) \mathrm{d} x, \quad n \geq 2 .
$$

2. We have $\mathrm{E} \tau_{y}=m_{1}(y)$, so that $m(y)=m_{1}(y)$, and $\operatorname{var} \tau_{y}=m_{1}(y)^{2}-2 m_{2}(y)$.

3. For all $s \geq 0$, there exists a unique solution $\psi(s, \cdot)$ in $\mathcal{M}_{\mathrm{abs}}$ of the equation $\mathcal{A} \psi(s, x)=$ $s \psi(s, x)$ with initial condition $\psi(s, 0)=1$. Moreover,

$$
\psi(s, y)=1+s \int_{0}^{y}\left(1+\int_{0}^{x} R_{s}(x, u) \mathrm{d} u\right) \mathrm{d} x .
$$

4. The Laplace transform of $\tau_{y}$ is given by $\mathrm{E}^{-s \tau_{y}}=1 / \psi(s, y)$.

Proof. A generator equation $\mathcal{A} f(x)=z(x)$ with $z \in \mathcal{M}_{\text {abs }}$ can be written as an integral equation for the density $f^{\prime}$, namely,

$$
f^{\prime}(x)=z(x)+\int_{0}^{x} K(x, y) f^{\prime}(y) \mathrm{d} y,
$$

where $K(x, y):=K_{0}(x, y)=\lambda(x) \mu_{x}(y)$. Similarly, the equation

$$
\mathcal{A} f(x)=s f(x), \quad f(0)=1,
$$

is equivalent to

$$
f^{\prime}(x)=s f(x)+\int_{0}^{x} K(x, y) f^{\prime}(y) \mathrm{d} y=s+\int_{0}^{x} K_{s}(x, y) f^{\prime}(y) \mathrm{d} y .
$$

It is well known that a solution of (9) is given by

$$
f^{\prime}(x)=z(x)+\int_{0}^{x} R_{0}(x, y) z(y) \mathrm{d} y,
$$

and that (10) is solved by

$$
f^{\prime}(x)=s\left(1+\int_{0}^{x} R_{s}(x, y) \mathrm{d} y\right) .
$$

Note that certainly $f \in \mathcal{M}_{\text {abs }}$, since $f$ is absolutely continuous and $f^{\prime}$ is locally bounded and nonnegative. The homogeneous equation

$$
h^{\prime}(x)=\int_{0}^{x} K_{s}(x, y) h^{\prime}(y) \mathrm{d} y
$$


is solved in the set of absolutely continuous functions only by constant functions $h$. This is immediate from the fact that iteration yields

$$
\left|h^{\prime}(x)\right|=\left|\int_{0}^{x} K_{s}^{n}(x, y) h^{\prime}(y) \mathrm{d} y\right| \leq(\lambda(x)+s) \int_{0}^{x} \frac{\left(\int_{y}^{x}(\lambda(u)+s) \mathrm{d} u\right)^{n-1}}{(n-1) !}\left|h^{\prime}(y)\right| \mathrm{d} y
$$

for all $n \in \mathbb{N}$ and, hence, $h^{\prime}(x)=0$. Consequently, solutions of (9) and (10) are unique in $\mathcal{M}_{\text {abs }}$, once we specify $f(0)$.

Since $\mathcal{A} m_{1}(x)=1$, it follows that the process $U_{1, t}=m_{1}\left(X_{t}\right)-t$ is a martingale. Now, since $\mathrm{E} \tau_{y}<\infty$, then on $\left\{\tau_{y}>t\right\}$ we have $\left|U_{1, t}\right| \leq t+m_{1}\left(X_{\tau_{y}}\right) \leq t+m_{1}(y) \leq \tau_{y}+m_{1}(y)$, so that

$$
\mathrm{E}\left(\left|U_{1, t}\right| ; \tau_{y}>t\right) \leq \mathrm{E}\left(\tau_{y} ; \tau_{y}>t\right)+m_{1}(y) \mathrm{P}\left(\tau_{y}>t\right)=o(1) \quad \text { as } t \rightarrow \infty,
$$

since $\mathrm{E} \tau_{y}<\infty$. This justifies optional stopping for the martingale $U_{1, t}$ at time $\tau_{y}$ (see [15]) and it follows from $m_{1}(0)=0$ that $m_{1}(y)=m(y)=\mathrm{E} \tau_{y}$.

The integrated process $I_{t}=\int_{0}^{t} s \mathrm{~d}\left(m\left(X_{s}\right)-s\right)$ is also a martingale (see again [15, p. 92]) and it follows by partial integration that

$$
I_{t}=t m\left(X_{t}\right)-\frac{1}{2} t^{2}-m_{2}\left(X_{t}\right)+D_{t}
$$

where $D_{t}=m_{2}\left(X_{t}\right)-\int_{0}^{t} m\left(X_{s}\right) \mathrm{d} s$ is the Dynkin martingale of the function $m_{2}$. Hence, the difference

$$
U_{2, t}=\operatorname{tm}\left(X_{t}\right)-\frac{1}{2} t^{2}-m_{2}\left(X_{t}\right)
$$

of $I_{t}$ and $D_{t}$ is a martingale too. Optional stopping, which can be justified as in (11), leads to

$$
\operatorname{var} \tau_{y}=\mathrm{E} \tau_{y}^{2}-\left(\mathrm{E} \tau_{y}\right)^{2}=m(y)^{2}-2 m_{2}(y)
$$

showing part 2. We now turn to the function $\psi$. Since $\mathcal{A} \psi(s, x)=s \psi(s, x)$ with $\psi(0, x)=1$, we have

$$
\psi^{\prime}(s, x)=s+\int_{0}^{x} K_{s}(x, y) \psi^{\prime}(s, y) \mathrm{d} y,
$$

which is tantamount to (10). Following the discussion above we conclude that a unique solution in $\mathcal{M}_{\text {abs }}$ exists and $\psi(s, \cdot)$ is given in terms of the associated resolvent kernel as in (8), so that part 3 is proved.

It is known that the process $\mathrm{e}^{-s t} \psi\left(s, X_{t}\right)$ is a martingale (see, e.g. [15, p. 175] or [24]). Optional stopping at $\tau_{y}$, which can be justified as in [19], leads to $\mathrm{E}^{-s \tau_{y}} \psi(s, y)=\psi(s, 0)=1$, so that part 4 is proved.

Remark. For $n=1$, we can prove the equality $\mathcal{A} m(x)=1$ by an alternative probabilistic reasoning, avoiding the use of martingales. The equation to be solved becomes

$$
m^{\prime}(y)=1+\lambda(y) \int_{0}^{y} m^{\prime}(u) \mu_{y}(u) \mathrm{d} u .
$$

Consider the first jump time $T_{1}$. If $T_{1} \geq y$ then $\tau_{y}=y$, while if $T_{1}<y$ then $\tau_{y}$ is equal to $T_{1}$ plus the hitting time of $y$, starting at $X_{T_{1}}$. Hence,

$$
\tau_{y} \stackrel{\mathrm{D}}{=} y \mathbf{1}_{\left\{T_{1} \geq y\right\}}+\left(T_{1}+\tau_{y}^{\prime}-\tau_{X_{T_{1}}}^{\prime}\right) \mathbf{1}_{\left\{T_{1}<y\right\}}
$$


where $\tau^{\prime}$ is the family of hitting times for a process $X^{\prime} \stackrel{\mathrm{D}}{=} X$. that is independent of $X$.. It follows that

$$
m(y)=y+\frac{\mathrm{E}\left(T_{1}-\tau_{X_{T_{1}}}^{\prime} ; T_{1}<y\right)}{\mathrm{P}\left(T_{1} \geq y\right)} .
$$

Conditioning on $T_{1}$ yields

$$
m(y)=y+\frac{\int_{0}^{y}\left(t-\int_{0}^{t} m(u) \mathrm{d} \mu_{t}(u)\right) \mathrm{P}\left(T_{1} \in \mathrm{d} t\right)}{\mathrm{P}\left(T_{1} \geq y\right)} .
$$

Using $\mathrm{dP}\left(T_{1} \leq y\right) / \mathrm{d} y=\lambda(y) \mathrm{P}\left(T_{1} \geq y\right)$, we obtain (12) after a short calculation.

Theorem 2. We have the power series representation

$$
\psi(s, x)=\sum_{n=0}^{\infty} m_{n}(x) s^{n}
$$

for all $s \geq 0$ and all $x \geq 0$.

Proof. To show (13), we first prove by induction that $m_{n}(y) \leq m(y)^{n} / n$ !, which is certainly true for $n=1$. Moreover, if the assumption holds for $n-1$ then

$$
\begin{aligned}
m_{n}(y) & =\int_{0}^{y}\left(m_{n-1}(x)+\int_{0}^{x} R_{0}(x, u) m_{n-1}(u) \mathrm{d} u\right) \mathrm{d} x \\
& \leq \int_{0}^{y} m_{n-1}(x) m^{\prime}(x) \mathrm{d} x \\
& \leq \int_{0}^{y} \frac{m(x)^{n-1}}{(n-1) !} m^{\prime}(x) \mathrm{d} x \\
& =\frac{m(x)^{n}}{n !} .
\end{aligned}
$$

It follows that the series in (13) converges for all $s \geq 0$ and all $x \geq 0$. The function

$$
h(x):=\sum_{n=0}^{\infty} s^{n} m_{n}(x) \leq \mathrm{e}^{s m(x)}
$$

is in $\mathcal{M}_{\mathrm{abs}}$ and $h_{k}(x):=\sum_{n=0}^{k} s^{n} m_{n}(x)$ converges to $h(x)$ pointwise as $k \rightarrow \infty$. Since

$$
\mathcal{A} h_{k}(x)=\sum_{n=1}^{k} s^{n} m_{n-1}(x)=s \sum_{n=0}^{k-1} s^{n} m_{n}(x)=s h_{k}(x)-s^{k} m_{k}(x),
$$

it follows that

$$
\left|\mathcal{A} h_{k}(x)-s h_{k}(x)\right| \leq \frac{(\operatorname{sm}(x))^{k}}{k !} .
$$

In particular, $\mathcal{A} h_{k}(x)-s h_{k}(x)$ tends to 0 as $k \rightarrow \infty$. Hence, $\mathcal{A} h(x)=\operatorname{sh}(x)$ and, by the uniqueness property, $\psi(s, x)=h(x)$.

The following corollary is needed in the next section. 
Corollary 1. The function

$$
s \mapsto \frac{\psi(s, y)-1}{s}
$$

is increasing for all $y \in[0, \infty)$ and in particular $\psi(s, y) \geq 1+\operatorname{sm}(y)$.

Proof. We have

$$
\frac{\psi(s, y)-1}{s}=\sum_{n=1}^{\infty} m_{n}(y) s^{n-1}
$$

The next theorem gives a sufficient criterion for $\tau_{y} / m(y)$ to be asymptotically exponential without the assumption of ergodicity.

Theorem 3. If $m_{2}(y)=o\left(m(y)^{2}\right)$ then $\tau_{y} / m(y) \stackrel{\mathrm{w}}{\rightarrow} Z$ as $y \rightarrow \infty$.

Proof. We carry out an induction proof to show that $m_{n}(y)=o\left(m(y)^{n}\right)$ for all $n \geq 2$. We have $m_{2}(y)=o\left(m(y)^{2}\right)$ by assumption. If the assertion is true for $n-1$, we obtain, using representation (7) and the monotonicity of the functions $m_{n}(y)$,

$$
\begin{aligned}
m_{n}(y) & =\int_{0}^{y}\left(m_{n-1}(x)+\int_{0}^{x} R_{0}(x, u) m_{n-1}(u) \mathrm{d} u\right) \mathrm{d} x \\
& \leq m_{n-1}(y) \int_{0}^{y}\left(1+\int_{0}^{x} R_{0}(x, u) \mathrm{d} u\right) \mathrm{d} x \\
& =o\left(m^{n-1}(y)\right) m(y) \\
& =o\left(m(y)^{n}\right) .
\end{aligned}
$$

It now follows from Theorem 1 that

$$
\lim _{y \rightarrow \infty} \mathrm{E}^{-s \tau_{y} / m(y)}=\lim _{y \rightarrow \infty} \psi\left(\frac{s}{m(y)} y^{-1}\right)=\lim _{y \rightarrow \infty}\left(\sum_{n=0}^{\infty} s \frac{m_{n}(y)}{m(y)^{n}}\right)^{-1} .
$$

Since $\sup _{y} m_{n}(y) / m(y)^{n} \leq 1 / n !$ and $\lim _{y \rightarrow \infty} m_{n}(y) / m(y)^{n}=0$ for all $n \geq 2$, we can use Lebesgue's convergence theorem and conclude that the right-hand side of (14) tends to $1 /(1+s)$ as $y \rightarrow \infty$, i.e. to the Laplace transform of $Z$. This completes the proof.

\section{Asymptotics of the running maximum}

We now consider the asymptotic behavior of $M_{t}$ in two cases: (i) $m(x)$ is regularly varying and (ii) $m(x)$ is rapidly varying. Assuming ergodicity, case (i) is a straightforward consequence of known results. Case (ii) is more complicated.

Theorem 4. If $m \in \mathcal{R}_{a}$ for some $a \in(0, \infty)$ and $X_{t}$ is ergodic, then

$$
\frac{M_{t}}{m^{-1}(t)} \stackrel{\mathrm{w}}{\rightarrow} Z_{a} \quad \text { as } t \rightarrow \infty
$$

Proof. If $m \in \mathcal{R}_{a}$, it follows from (2) that $1-G \in \mathcal{R}_{-a}$. Hence, the conditions for convergence in (4) are satisfied. Since $\vec{G}$ is increasing and unbounded, Theorem A of [12] implies that $\vec{G}(t) \sim m^{-1}\left(\mu_{c} t\right)$, yielding $M_{t} / m^{-1}(t) \stackrel{\mathrm{W}}{\rightarrow} Z_{a}$ as $t \rightarrow \infty$. 
Theorem 5. If $m \in \mathcal{R}_{\infty}$ then

$$
\mathrm{E}\left(\left(\frac{M_{t}}{m^{-1}(t)}\right)^{n}\right) \rightarrow 1 \quad \text { as } t \rightarrow \infty
$$

for all $n \geq 0$. In particular,

$$
\frac{M_{t}}{m^{-1}(t)} \stackrel{\mathrm{w}}{\rightarrow} 1 \text { as } t \rightarrow \infty .
$$

Proof. We define $\kappa_{n}(t)=\mathrm{E} M_{t}^{n}$ and show that

$$
\left(m^{-1}\left(\frac{1}{s}\right)\right)^{-n} \int_{0}^{\infty} \mathrm{e}^{-s t} \mathrm{~d} \kappa_{n}(t) \rightarrow 1 \quad \text { as } s \rightarrow 0
$$

for every $n \in \mathbb{N}$. By Karamata's Tauberian theorem, (17) implies that $\mathrm{E}\left(M_{t}^{n}\right) \sim\left(m^{-1}(t)\right)^{n}$ as $t \rightarrow \infty$, and, hence, we have proved (15). Since the constant moment sequence obviously satisfies Carleman's criterion, (16) follows immediately.

To prove (17), let $y=m^{-1}(1 / s)$. Then

$$
\begin{aligned}
y^{-n} \int_{0}^{\infty} \mathrm{e}^{-s t} \mathrm{~d} \kappa_{n}(t) & =y^{-n} \int_{0}^{\infty} \mathrm{e}^{-s t} \frac{\mathrm{d}}{\mathrm{d} t}\left(\mathrm{E} M_{t}^{n}\right) \mathrm{d} t \\
& =y^{-n} \int_{0}^{\infty} \mathrm{e}^{-s t} \frac{\mathrm{d}}{\mathrm{d} t}\left(\int_{0}^{\infty} n u^{n-1} \mathrm{P}\left(M_{t}>u\right) \mathrm{d} u\right) \mathrm{d} t \\
& =y^{-n} \int_{0}^{\infty} n u^{n-1} \int_{0}^{\infty} \mathrm{e}^{-s t} \mathrm{P}\left(\tau_{u} \in \mathrm{d} t\right) \mathrm{d} u \\
& =y^{-n} \int_{0}^{\infty} \frac{n u^{n-1}}{\psi(s, u)} \mathrm{d} u \\
& =J_{0}^{\infty}(y)
\end{aligned}
$$

where we define

$$
J_{a}^{b}(y):=\int_{a}^{b} \frac{n u^{n-1}}{\psi(1 / m(y), y u)} \mathrm{d} u .
$$

We show that $J_{0}^{\infty}(y) \rightarrow 1$ as $y \rightarrow \infty$ by dividing the range of integration into three parts.

$\operatorname{Part}(i): J_{w}^{\infty}(y) \rightarrow 0$ for any $w>1$. According to Corollary 1 we have $\psi(s, y) \geq 1+\operatorname{sm}(y)$. Hence,

$$
\psi\left(\frac{1}{m(y)}, u y\right) \geq 1+\frac{m(u y)}{m(y)}=1+\frac{r(u y)}{r(y)} u^{n+1},
$$

where $r(x):=x^{n+1} m(x)$ is again rapidly varying. The convergence $r(u y) / r(y) \rightarrow \infty$ is uniform for $u \geq w>1$ (see (27) in Appendix A). In particular, for all $K>0$, we ultimately have $\inf _{u \geq w} r(u y) / r(y) \geq K$ for large $y$, yielding

$$
J_{w}^{\infty}(y) \leq \int_{w}^{\infty} \frac{n u^{n-1}}{1+[r(u y) / r(y)] u^{n+1}} \mathrm{~d} u \leq \int_{w}^{\infty} \frac{n u^{n-1}}{1+K u^{n+1}} \mathrm{~d} u
$$

for sufficiently large $t$, so that $J_{w}^{\infty}(y)$ tends to 0 as $y \rightarrow \infty$.

Part (ii): $J_{1}^{w}(y) \rightarrow 0$ for any $w>1$. This is clear, since the integrand tends to 0 and is uniformly bounded by $n w^{n-1}$ on the bounded interval $[1, w]$. 
Part (iii): $J_{0}^{1}(y) \rightarrow 1$. Let $u \in(0,1)$ and choose an $\varepsilon>0$. Since $m_{n}(y) / m(y)^{n} \leq 1 / n$ ! and $m(y u) / m(y) \rightarrow 0$ as $y \rightarrow \infty$, it follows that

$$
\begin{aligned}
\limsup _{y \rightarrow \infty} \psi\left(\frac{s}{m(y)}, y u\right) & =\limsup _{y \rightarrow \infty} \psi\left(\frac{s}{m(y u)} \frac{m(y u)}{m(y)}, y u\right) \\
& \leq \limsup _{y \rightarrow \infty} \psi\left(\frac{\varepsilon}{m(y u)}, y u\right) \\
& =\limsup _{y \rightarrow \infty} \sum_{n=0}^{\infty} \varepsilon^{n} \frac{m_{n}(y u)}{m(y u)^{n}} \\
& \leq \mathrm{e}^{\varepsilon} .
\end{aligned}
$$

On the other hand, again using Corollary 1 ,

$$
\liminf _{y \rightarrow \infty} \psi\left(\frac{s}{m(y)}, y u\right) \geq \liminf _{y \rightarrow \infty}\left(1+s \frac{m(y u)}{m(y)}\right)=1 .
$$

Since $\varepsilon$ was arbitrary, it follows that $J_{0}^{1}(y) \rightarrow \int_{0}^{1} n u^{n-1} \mathrm{~d} u=1$.

\section{Applications to special cases}

We have seen that $m(y)=\mathrm{E}\left(\tau_{y}\right)$ serves as a normalizing function in (3) (in the ergodic case) and in Theorem 3, while its inverse $m^{-1}(t)$ plays a similar role in Theorems 4 and 5 . Therefore, explicit formulae for these functions are of special interest. In several examples we can compute $m(y)$ via the unique solution in $\mathcal{M}_{\text {abs }}$ of the integral equation given in Theorem 1 for $n=1$, which reads as

$$
m^{\prime}(y)=1+\lambda(y) \int_{0}^{y} m^{\prime}(u) \mu_{y}(u) \mathrm{d} u .
$$

Similarly, we can solve the equations

$$
\begin{aligned}
\psi^{\prime}(s, y) & =s \psi(s, y)+\lambda(y) \int_{0}^{y} \psi^{\prime}(s, u) \mu_{y}(u) \mathrm{d} u \\
m_{2}^{\prime}(y) & =m(y)+\lambda(y) \int_{0}^{y} m_{2}^{\prime}(u) \mu_{y}(u) \mathrm{d} u
\end{aligned}
$$

in $\mathcal{M}_{\text {abs }}$ to find $\mathrm{Ee}^{-s \tau_{y}}=1 / \psi(s, y)$ and var $\tau_{y}=m_{1}(y)^{2}-2 m_{2}(y)$. Let us consider a few examples.

\subsection{Separable jump measures}

Suppose that the jump measures $\mu_{x}$ are given in the form

$$
\mu_{x}(y)=\frac{v(y)}{v(x)}, \quad x \geq y \geq 0,
$$

for some nondecreasing function $v:[0, \infty) \rightarrow[0, \infty)$ (defining $0 / 0$ as 0 ). We give some examples at the end of this subsection. 
Theorem 6. For an MGCP with $\mu_{x}(y)=v(x) / v(y)$ as above and general intensity function $\lambda(x)$, the mean of the first hitting time $\tau_{y}$ is given in closed form by

$$
m(y)=y+\int_{0}^{y} \frac{\lambda(x)}{v(x)} \int_{0}^{x} v(w) \exp \left(\int_{w}^{x} \lambda(v) \mathrm{d} v\right) \mathrm{d} w \mathrm{~d} x .
$$

The variance of $\tau_{y}$ can be computed from (19) and

$$
m_{2}(y)=\int_{0}^{y}\left[m(x)+\frac{\lambda(x)}{v(x)} \int_{0}^{x} m(w) v(w) \exp \left(\int_{w}^{x} \lambda(v) \mathrm{d} v\right) \mathrm{d} w\right] \mathrm{d} x .
$$

Proof. Let $A \in \mathcal{M}_{\text {abs }}$ be arbitrary, and define $z(x)=\int_{0}^{x} z^{\prime}(u) \mathrm{d} u$ by setting

$$
z^{\prime}(y):=A(y)+\frac{\lambda(y)}{v(y)} \int_{0}^{y} A(w) v(w) \exp \left(\int_{w}^{y} \lambda(v) \mathrm{d} v\right) \mathrm{d} w .
$$

A straightforward calculation yields

$$
\begin{aligned}
\int_{0}^{y} v(u)\left(z^{\prime}(u)-A(u)\right) \mathrm{d} u & =\int_{0}^{y} \lambda(u) \int_{0}^{u} A(w) v(w) \exp \left(\int_{w}^{u} \lambda(v) \mathrm{d} v\right) \mathrm{d} w \mathrm{~d} u \\
& =\int_{0}^{y} A(w) v(w) \int_{w}^{y} \lambda(u) \exp \left(\int_{w}^{u} \lambda(v) \mathrm{d} v\right) \mathrm{d} u \mathrm{~d} w \\
& =\int_{0}^{y} A(w) v(w)\left(\exp \left(\int_{w}^{y} \lambda(v) \mathrm{d} v\right)-1\right) \mathrm{d} w \\
& =\int_{0}^{y} A(w) v(w) \exp \left(\int_{w}^{y} \lambda(v) \mathrm{d} v\right) \mathrm{d} w-\int_{0}^{y} A(u) v(u) \mathrm{d} u \\
& =\frac{v(y)}{\lambda(y)}\left(z^{\prime}(y)-A(y)\right)-\int_{0}^{y} A(u) v(u) \mathrm{d} u .
\end{aligned}
$$

Hence,

$$
A(y)=z^{\prime}(y)-\lambda(y) \int_{0}^{y} z^{\prime}(u) \frac{v(u)}{v(y)} \mathrm{d} u .
$$

Letting $A(y)=1$ and $A(y)=m(y)$ we obtain (19) and (20), respectively.

Regarding the Laplace transform of $\tau_{y}$, the required solution to $\mathcal{A} f(x)=s f(x)$ does not seem easy to find. If all functions involved are smooth enough, we can transform the generator equation into

$$
\frac{\partial^{2}}{\partial x^{2}} \psi(s, x)-(s+\xi(x)+\lambda(x)) \psi^{\prime}(s, x)+s \xi(x) \psi(s, x)=0, \quad \psi(0, x)=1,
$$

where

$$
\xi(x)=\frac{\lambda^{\prime}(x)}{\lambda(x)}-\frac{v^{\prime}(x)}{v(x)} .
$$

Fixing $s$ and defining $h(x)$ by $\psi(s, x)=\mathrm{e}^{h(x)}$ we arrive at the Riccati equation

$$
h^{\prime 2}(x)+h^{\prime \prime}(x)-(s+\xi(x)+\lambda(x)) h^{\prime}(x)+s \xi(x)=0, \quad h^{\prime}(0)=s,
$$

which is difficult to solve in general.

Now we turn to the running maximum. For regularly varying $v(x)$, we have the following result. 
Theorem 7. Suppose that

$$
\lim _{x \rightarrow \infty} x \lambda(x)=a \in(1, \infty]
$$

and that $v \in \mathcal{R}_{b}$ for some $b<a-1$.

(a) If $a<\infty$ then

$$
\frac{M_{t}}{m^{-1}(t)} \stackrel{\mathrm{w}}{\rightarrow} Z_{a-b} \quad \text { as } t \rightarrow \infty .
$$

(b) If $a=\infty$ then $M_{t} / m^{-1}(t) \stackrel{\mathrm{w}}{\rightarrow} 1$ as $t \rightarrow \infty$.

Proof. We have $b \geq 0$ because $v(x)$ is nondecreasing. By Proposition 3 of [8], $X_{t}$ is ergodic if $\lim \sup _{x \rightarrow \infty} \lambda(x) \int_{0}^{x} \mu_{x}(y) \mathrm{d} y>1$. In our case, for $a<\infty$, this lim sup is given by

$$
\begin{aligned}
\limsup _{x \rightarrow \infty} \lambda(x) \int_{0}^{x} \frac{v(y)}{v(x)} \mathrm{d} y & =\limsup _{x \rightarrow \infty} \frac{a}{x v(x)} \int_{0}^{x} v(y) \mathrm{d} y \\
& =\limsup _{x \rightarrow \infty} \frac{a}{x v(x)} \frac{x v(x)}{b+1} \\
& =\frac{a}{b+1} \\
& >1
\end{aligned}
$$

(where we have used part 1 of Theorem 8 in Appendix A for the second equality), and, for $a=\infty$, it is infinite. By Theorems 4 and 5, it remains to show that $m \in \mathcal{R}_{a-b}$. This is done in the following lemma (in which no inequality between $a$ and $b$ is assumed).

Lemma 1. Suppose that $v \in \mathcal{R}_{b}$ for some $b<\infty$ and that $x \lambda(x) \rightarrow a \in(0, \infty)$. Then $m \in \mathcal{R}_{1 \vee(a-b)}$.

Proof. If $x \lambda(x) \rightarrow a$ then $\lambda \in \mathcal{R}_{-1}$ and it follows that

$$
x \mapsto \frac{\lambda(x)}{v(x)} \exp \left(\int_{0}^{x} \lambda(s) \mathrm{d} s\right) \in \mathcal{R}_{a-1-b} .
$$

If $b-a \geq-1$ then, by Theorem 9 in Appendix A,

$$
x \mapsto \frac{\lambda(x) \exp \left(\int_{0}^{x} \lambda(s) \mathrm{d} s\right)}{v(x)} \int_{0}^{x} v(u) \exp \left(-\int_{0}^{u} \lambda(s) \mathrm{d} s\right) \mathrm{d} u \in \mathcal{R}_{0},
$$

yielding $m \in \mathcal{R}_{1}$. If $b-a<-1$ then $\int_{0}^{\infty} v(u) \exp \left(-\int_{0}^{u} \lambda(s) \mathrm{d} s\right) \mathrm{d} u<\infty$,

$$
x \mapsto \frac{\lambda(x) \exp \left(\int_{0}^{x} \lambda(s) \mathrm{d} s\right)}{v(x)} \int_{0}^{x} v(u) \exp \left(-\int_{0}^{u} \lambda(s) \mathrm{d} s\right) \mathrm{d} u \in \mathcal{R}_{-1-b+a},
$$

and $m \in \mathcal{R}_{a-b}$. Note that $a-b>1$ implies that $y / m(y) \rightarrow 0$. If $a=\infty$ then $x \mapsto v(x) \exp \left(-\int_{0}^{x} \lambda(s) \mathrm{d} s\right)$ is slowly varying and $x \mapsto \int_{0}^{x} v(u) \exp \left(-\int_{0}^{u} \lambda(s) \mathrm{d} s\right) \mathrm{d} u$ is in $\mathcal{R}_{1}$. Thus, $m \in \mathcal{R}_{\infty}$.

Example 1. (Renewal age processes.) If $v(x) \equiv 1$ then $\mu_{x}(y) \equiv 1$, i.e. the process restarts at 0 after each jump. This is the age process from renewal theory, where the renewal epochs have a distribution with density $x \mapsto \lambda(x) \exp \left(-\int_{0}^{x} \lambda(u) \mathrm{d} u\right), x \geq 0$. Note that $\tau_{y}$ is the first 
time at which the current lifetime reaches $y$. Equations (19) and (20) yield, after some further calculations,

$$
m(y)=\int_{0}^{y} \exp \left(\int_{w}^{y} \lambda(v) \mathrm{d} v\right) \mathrm{d} w, \quad m_{2}(y)=\int_{0}^{y}(y-w) \exp \left(\int_{w}^{y} \lambda(v) \mathrm{d} v\right) \mathrm{d} w .
$$

The Laplace transform of $\tau_{y}$ is given by

$$
\mathrm{Ee}^{-s \tau_{y}}=\left(1+s \int_{0}^{y} \exp \left(\int_{u}^{y}(\lambda(w)+s) \mathrm{d} w\right) \mathrm{d} u\right)^{-1} .
$$

This follows immediately from (18) which reads as

$$
\psi^{\prime}(s, y)=s \psi(s, y)+\lambda(y)(\psi(s, y)-1) .
$$

The case where $\lambda(x) \equiv \lambda$ is constant has been discussed in [26].

Example 2. (Coupled intensity rate and jump measure.) Let $v(x)=\lambda(x)$ for all $x \geq 0$. Then we obtain, from (19),

$$
m(y)=\int_{0}^{y} \exp \left(\int_{0}^{x} \lambda(w) \mathrm{d} w\right) \mathrm{d} x .
$$

Moreover, it follows from (21) that

$$
\frac{\partial^{2}}{\partial x^{2}} \psi(s, x)=(s+\lambda(x)) \psi^{\prime}(s, x)
$$

and, thus, $\psi^{\prime}(s, x)=s \exp \left(s x+\int_{0}^{x} \lambda(w) \mathrm{d} w\right)$. Hence,

$$
\psi(s, y)=1+s \int_{0}^{y} \exp \left(s x+\int_{0}^{x} \lambda(s) \mathrm{d} s\right) \mathrm{d} x
$$

and, thus,

$$
\mathrm{Ee}^{-s \tau_{y}}=\left(1+s \int_{0}^{y} \exp \left(s x+\int_{0}^{x} \lambda(s) \mathrm{d} s\right) \mathrm{d} x\right)^{-1} .
$$

This generalizes the result for the particular case $\lambda(x)=\lambda x$ and $\mu_{x}(y)=y / x$ in [8].

\subsection{MGCPs with multiplicative jumps}

Consider the case where at each jump time the current level of the process is multiplied by an independent random variable $Q$ having a distribution function $F$ whose support is contained in $[0,1)$ (i.e. $F(1-)=1$ ). Owing to their importance in applications, these MGCPs have been frequently studied [1], [4], [9], [13], [16], [20], [21], [22], [23]. Clearly, $\mu_{y}(u)=F(u / y)$, and if we assume that $\lambda(x) \equiv \lambda$ then

$$
\begin{aligned}
m^{\prime}(y) & =1+\lambda \int_{0}^{y} m^{\prime}(u) F\left(\frac{u}{y}\right) \mathrm{d} u, \\
\psi^{\prime}(s, y) & =s \psi(s, y)+\lambda \int_{0}^{y} \psi^{\prime}(s, u) F\left(\frac{u}{y}\right) \mathrm{d} u .
\end{aligned}
$$

Suppose that $m(\cdot)$ and $\psi(s, \cdot)$ can be expanded into power series: $m(x)=\sum_{k=1}^{\infty} a_{k} x^{k}$ and $\psi(s, x)=\sum_{k=1}^{\infty} b_{k} x^{k}$. Then, letting $\theta_{k}=1-\mathrm{E} Q^{k}$,

$$
1=\sum_{k=1}^{\infty} a_{k} k x^{k-1}-\lambda \sum_{k=1}^{\infty} \theta_{k} a_{k} x^{k}=\sum_{k=1}^{\infty}\left(a_{k+1}(k+1)-\lambda \theta_{k} a_{k}\right) x^{k} .
$$


Hence,

$$
a_{k+1}=\lambda \frac{\theta_{k}}{k+1} a_{k}, \quad a_{1}=m^{\prime}(0)=1,
$$

yielding

$$
m(x)=\frac{1}{\lambda} \sum_{k=1}^{\infty} \frac{\prod_{i=1}^{k-1} \theta_{i}}{k !}(\lambda x)^{k} .
$$

Similarly, the power series of $\psi(s, x)$ satisfies

$$
s \sum_{k=1}^{\infty} b_{k} x^{k}=\sum_{k=1}^{\infty}\left(b_{k+1}(k+1)-\lambda \theta_{k} b_{k}\right) x^{k},
$$

which means that

$$
b_{k+1}=\frac{\lambda \theta_{k}+s}{k+1} b_{k}, \quad b_{0}=\psi(s, 0)=1,
$$

and, therefore, leads to

$$
\psi(s, x)=1+s \sum_{k=1}^{\infty} \frac{\prod_{i=1}^{k-1}\left(\lambda \theta_{i}+s\right)}{k !} x^{k} .
$$

The two power series in (24) and (25) obviously have infinite radius of convergence and satisfy (22) and (23), respectively, so that they are indeed the desired solutions.

Two special cases. (a) The collapse consists of a multiplication by a deterministic constant $q \in[0,1)$, i.e. $F(x)=\mathbf{1}_{\{x \geq q\}}$. Then $\theta_{a}=1-q^{a}$ and, using the $q$-series symbols

$$
(q)_{k}=\prod_{i=1}^{k}\left(1-q^{i}\right) \quad \text { and } \quad(c ; q)_{k}=\prod_{i=0}^{k-1}\left(1-c q^{i}\right),
$$

we obtain

$$
m(x)=\frac{1}{\lambda} \sum_{k=1}^{\infty} \frac{(q)_{k-1}}{k !}(\lambda x)^{k}
$$

and

$$
\psi(s, x)=1+\sum_{k=1}^{\infty} \frac{(\lambda+s)^{k}(\lambda /(\lambda+s) ; q)_{k}}{k !} x^{k} .
$$

(b) $Q=U^{1 / \alpha}$ for some $\alpha>0$ and a uniform random variable $U$ on $(0,1)$. Then

$$
m(x)=\alpha \lambda^{-1} \int_{0}^{\lambda x} u^{-\alpha} \mathrm{e}^{u} \int_{0}^{u} t^{\alpha-1} \mathrm{e}^{-t} \mathrm{~d} t \mathrm{~d} u
$$

and

$$
\psi(s, x)=H\left(\alpha \frac{s}{s+\lambda}, \alpha ;(\lambda+s) x\right),
$$

where

$$
H(a, b ; x)=\sum_{k=0}^{\infty} \frac{(a)_{k}}{(b)_{k}} \frac{x^{k}}{k !}
$$

is the standard hypergeometric function. 


\subsection{The Cramér-Lundberg model in risk theory}

The classical risk-reserve process in the Cramér-Lundberg model is given by

$$
R_{t}=t-\sum_{k=1}^{N_{t}} B_{k}
$$

where the claims $B_{k}$ are independent, have a common distribution function $B$, and $N_{t}$ is a Poisson process with intensity $\lambda$ which is independent of the $B_{k}$. Let $\underline{R}_{t}=\inf _{s \leq t} R_{s}$, and consider the reflected process

$$
X_{t}=R_{t}-\underline{R}_{t} \text {. }
$$

The process $X_{t}$ can be interpreted as a risk-reserve process, where successive ruins are ignored. It is easy to see that $X_{t}$ is an MGCP with $\lambda(x)=\lambda$ and $\mu_{y}(u)=1-B(y-u-)$, and

$$
m^{\prime}(y)=1+\lambda \int_{0}^{y} m^{\prime}(u)(1-B(y-u)) \mathrm{d} u=1+\left(m^{\prime} * B_{I}\right)(y),
$$

where $B_{I}(x)=\lambda \int_{0}^{x}(1-B(u)) \mathrm{d} u$ and ' $*$ ' denotes the convolution operator. In what follows we write $\eta_{n}=\int_{0}^{\infty} u^{n} \mathrm{~d} B(u)$.

The above renewal equation has the unique solution

$$
m^{\prime}(x)=\sum_{k=0}^{\infty} B_{I}^{k *}(x)
$$

Therefore, the asymptotic behavior of $m(y)$ can be deduced from known results in renewal theory.

Theorem 8. 1. If $\lambda \eta_{1}=1$ and $\eta_{2}<\infty$, then $m(y) \sim y^{2} / \lambda \eta_{2}$.

2. If $1<\lambda \eta_{1}<\infty$ or $\eta_{1}=\infty$ and $\int_{0}^{\infty} \mathrm{e}^{-\delta x} \mathrm{~d} B_{I}(x)=1$ for some $\delta>0$, then

$$
m(y) \sim \frac{\mathrm{e}^{\delta y}}{\delta^{2} \int_{0}^{\infty} u \mathrm{e}^{-\delta u} \mathrm{~d} B_{I}(u)} .
$$

In this case we have

$$
\frac{M_{t}}{m^{-1}(t)} \stackrel{\mathrm{w}}{\rightarrow} 1
$$

3. If $\lambda \eta_{1}<1$ then $m(y) \sim y /\left(1-\lambda \eta_{1}\right)$. In addition, if there is a solution $\beta$ of the equation $\int_{0}^{\infty} \mathrm{e}^{\beta x} \mathrm{~d} B_{I}(x)=1$ with $\int_{0}^{\infty} u \mathrm{e}^{\beta u} \mathrm{~d} B_{I}(u)<\infty$, then $\left|y /\left(1-\lambda \eta_{1}\right)-m(y)\right|$ tends to a constant as $y \rightarrow \infty$.

Proof. The three cases follow from Propositions 6.1 and 7.2 and Theorem 7.1 of [3]. Note that

$$
\int_{0}^{\infty} u^{n} \mathrm{~d} B_{I}(u)=\lambda \int_{0}^{\infty} u^{n}(1-B(u)) \mathrm{d} u=\frac{\lambda}{n+1} \int_{0}^{\infty} u^{n+1} \mathrm{~d} B(u)=\frac{\lambda \eta_{n+1}}{n+1} .
$$

Regarding the Laplace transform of $\tau_{y}$, the equation for $\psi$ is given by

$$
\begin{aligned}
\psi^{\prime}(s, y) & =s \psi(s, y)+\lambda \int_{0}^{y} \psi^{\prime}(s, y)(u)(1-B(y-u)) \mathrm{d} u \\
& =s \psi(s, y)+\left(\psi(s, \cdot)^{\prime} * B_{I}\right)(y)
\end{aligned}
$$


and does not seem to be solvable in general. However, for the transform

$$
\Psi_{s}(t)=\int_{0}^{\infty} \mathrm{e}^{-t x} \psi(s, \mathrm{~d} x),
$$

there is a nice explicit formula in terms of the Laplace transform $\beta(t)=\int_{0}^{\infty} \mathrm{e}^{-t x} \mathrm{~d} B(x)$ of $B$. We obtain

$$
\Psi_{s}(t)=s \frac{\Psi_{s}(t)+1}{t}+\lambda \Psi_{s}(t) \frac{1-\beta(t)}{t} .
$$

Hence,

$$
\Psi_{s}(t)=\frac{s}{t-\lambda(1-\beta(t))-s} .
$$

\section{Appendix A. Regular and rapid variation}

A function $f:[0, \infty) \rightarrow(0, \infty)$ is regularly varying with index $a \in \mathbb{R}$ if, for all $\lambda>0$,

$$
\frac{f(\lambda y)}{f(y)} \rightarrow \lambda^{a} \text { as } y \rightarrow \infty \text {. }
$$

In this case we write $f \in \mathcal{R}_{a}$. The function $f$ is called rapidly varying if (26) holds with $a=\infty$ (letting $\lambda^{\infty}=0$ for $\lambda<1$ and $\lambda^{\infty}=\infty$ for $\lambda>1$ ) and slowly varying if (26) holds with $a=0$. The convergence in (26) is uniform for

$$
\lambda \in \begin{cases}{\left[c_{1}, \infty\right), c_{1}>0,} & a<0, \\ {\left[c_{1}, c_{2}\right], c_{1}>0,} & a=0, \\ \left(0, c_{2}\right], c_{2}>0, & a>0, \\ \left(0, c_{1}\right) \cup\left(c_{2}, \infty\right), c_{1}<1<c_{2}, & a=\infty\end{cases}
$$

where $c_{1}$ and $c_{2}$ are constants. The function $f$ is regularly varying with index $a<\infty$ if and only if $f$ is of the form

$$
f(x) \sim c \exp \left(\int_{1}^{x} U(w) \mathrm{d} w\right),
$$

where $c>0$ and $w U(w) \rightarrow a$. On the other hand, if

$$
f(x) \sim c(x) \exp \left(\int_{1}^{x} V(w) \mathrm{d} w\right),
$$

where $c(x)$ is nondecreasing and $w V(w) \rightarrow \infty$ as $w \rightarrow \infty$, then $f$ is rapidly varying. For $f$ to be rapidly varying, it is sufficient to show that $f(\lambda y) / f(y) \rightarrow \infty$ as $y \rightarrow \infty$ for all $\lambda>1$, or that

$$
\frac{x f^{\prime}(x)}{f(x)} \rightarrow \infty \quad \text { as } x \rightarrow \infty .
$$

If $f \in \mathcal{R}_{a}$ with index $a>0$ and is increasing, then its inverse, denoted by $f^{-1}$, is regularly varying with index $1 / a$ and vice versa, where we agree to understand $1 / \infty=0$ (see $[5$, Theorems 1.5.12 and 2.4.7]). Finally, Karamata's theorem (see [5, Section 1.6]) clarifies the behavior of the integral of a function in $\mathcal{R}_{a}$. 
Theorem 9. Let $f \in \mathcal{R}_{a}$ and $F(x)=\int_{0}^{x} f(w) \mathrm{d} w$.

1. If $f$ is locally bounded and $a>-1$, then $F(x) \sim x f(x) /(a+1)$.

2. If $a=-1$ and $x f(x)$ is locally integrable, then $x \mapsto \int_{0}^{x} f(u) \mathrm{d} u$ is slowly varying and $\int_{0}^{x} f(u) \mathrm{d} u /(x f(x)) \rightarrow \infty$. If, additionally, $\int_{0}^{\infty} f(u) \mathrm{d} u<\infty$ then $x \mapsto \int_{x}^{\infty} f(u) \mathrm{d} u<$ $\infty$ is slowly varying.

3. If $a<-1$ then $\int_{x}^{\infty} f(u) \mathrm{d} u<\infty$ for large $x$ and $\int_{x}^{\infty} f(u) \mathrm{d} u \sim x f(x) /|a+1|$.

4. If $a=\infty$ then $F \in \mathcal{R}_{\infty}$.

\section{Acknowledgement}

The authors are grateful to the anonymous referee whose careful reading of the paper and constructive comments led to several improvements.

\section{References}

[1] Altman, E., Avrachenkov, K., Kherani, A. A. and Prabhu, B. J. (2005). Performance analysis and stochastic stability of congestion control protocols. In INFOCOM '05, Vol. 2 (March 2005), pp. 1316-1327.

[2] Asmussen, S. (1998). Extreme value theory for queues via cycle maxima. Extremes 1, 137-168.

[3] Asmussen, S. (2003). Applied Probability and Queues, 2nd edn. Springer, New York.

[4] Bertoin, J. And Yor, M. (2005). Exponential functionals of Lévy processes. Prob. Surveys 2, 191-212.

[5] Bingham, N. H., Goldie, C. M. And Teugels, J. L. (1987). Regular Variation (Encyclopedia Math. Appl. 27). Cambridge University Press.

[6] Borovkov, K. And Last, G. (2008). On level crossings for a general class of piecewise-deterministic Markov processes. Adv. Appl. Prob. 40, 815-834.

[7] Borovkov, K. AND Vere-Jones, D. (2000). Explicit formulae for stationary distributions of stress release processes. J. Appl. Prob. 37, 315-321.

[8] Boxma, O., Perry, D., Stadje, W. and Zacks, S. (2006). A Markovian growth-collapse model. Adv. Appl. Prob. 38, 221-243.

[9] Carmona, P., Petit, F. And Yor, M. (2001). Exponential functionals of Lévy processes. In Lévy Processes, O. E. Barndorff-Nielsen et al. Birkhäuser, Boston, MA, pp. 41-55.

[10] Davis, M. H. A. (1984). Piecewise deterministic Markov processes: a general class of non-diffusion stochastic models. J. R. Statist. Soc. B 46, 353-388.

[11] Davis, M. H. A. (1993). Markov Models and Optimization (Monogr. Statist. Appl. Prob. 49). Chapman and Hall, London.

[12] Duurčić, D. And TorgašEv, A. (2007). Some asymptotic relations for the generalized inverse. J. Math. Anal. Appl. 335, 1397-1402.

[13] Dumas, V., Guillemin, F. and Robert, P. (2002). A Markovian analysis of additive-increase multiplicativedecrease algorithms. Adv. Appl. Prob. 34, 85-111.

[14] Eliazar, I. AND Klafter, J. (2004). A growth-collapse model: Lévy inflow, geometric crashes, and generalized Ornstein-Uhlenbeck dynamics. Physica A 334, 1-21.

[15] Ethier, S. N. and Kurtz, T. G. (1986). Markov Processes. John Wiley, New York.

[16] Guillemin, F., Robert, P. and Zwart, B. (2004). AIMD algorithms and exponential functionals. Ann. Appl. Prob. 14, 90-117.

[17] Kalashnikov, V. (1997). Geometric Sums: Bounds for Rare Events with Applications. Kluwer, Dordrecht.

[18] KeIlson, J. (1966). A limit theorem for passage times in ergodic regenerative processes. Ann. Math. Statist. 37, 866-870.

[19] Kella, O. and Stadje, W. (2001). On hitting times for compound Poisson dams with exponential jumps and linear release rate. J. Appl. Prob. 38, 781-786.

[20] Löpker, A. H. AND VAn LeeuwaARden, J. S. H. (2008). Transient moments of the TCP window size process. J. Appl. Prob. 45, 163-175.

[21] Maulik, K. And Zwart, B. (2006). Tail asymptotics for exponential functionals of Lévy processes. Stoch. Process. Appl. 116, 156-177.

[22] Ott, T. J. And Kemperman, J. H. B. (2008). Transient behavior of processes in the TCP paradigm. Prob. Eng. Inf. Sci. 22, 431-471. 
[23] OtT, T. J. AND SwAnson, J. (2007). Asymptotic behavior of a generalized TCP congestion avoidance algorithm. J. Appl. Prob. 44, 618-635.

[24] Palmowski, Z. ANd Rolski, T. (2002). A technique for exponential change of measure for Markov processes. Bernoulli 8, 767-785.

[25] Rootzén, H. (1988). Maxima and exceedances of stationary Markov chains. Adv. Appl. Prob. 20, 371-390.

[26] Van Leeuwaarden, J. S. H., Löpker, A. H. and Janssen, A. J. E. M. (2010). Connecting renewal age processes with M/D/1 and M/D/ $\infty$ queues through stick breaking. Stoch. Models 26, 141-163.

[27] Yafaev, D. R. (1985). On the asymptotics of solutions of Volterra integral equations. Ark. Mat. 23, $185-201$.

[28] Zheng, X. G. (1991). Ergodic theorems for stress release processes. Stoch. Process. Appl. 37, $239-258$. 